\title{
Mineral Phosphorus Supply in Piglets Impacts the Microbial Composition and Phytate Utilization in the Large Intestine
}

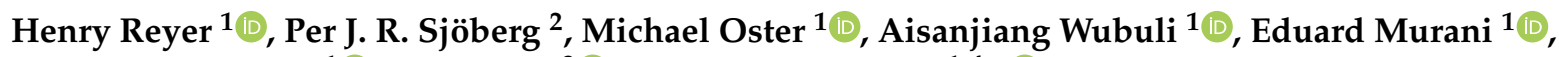 \\ Siriluck Ponsuksili ${ }^{1}$ (D) Petra Wolf ${ }^{3}$ (D) and Klaus Wimmers ${ }^{1,4, *(D)}$ \\ 1 Leibniz Institute for Farm Animal Biology (FBN), Institute for Genome Biology, Wilhelm-Stahl-Allee 2, \\ 18196 Dummerstorf, Germany; reyer@fbn-dummerstorf.de (H.R.); oster@fbn-dummerstorf.de (M.O.); \\ wubuli@fbn-dummerstorf.de (A.W.); murani@fbn-dummerstorf.de (E.M.); \\ ponsuksili@fbn-dummerstorf.de (S.P.) \\ 2 Department of Chemistry-BMC, Uppsala University, 75123 Uppsala, Sweden; Per.Sjoberg@kemi.uu.se \\ 3 Nutrition Physiology and Animal Nutrition, Faculty of Agricultural and Environmental Sciences, University \\ of Rostock, 18059 Rostock, Germany; petra.wolf@uni-rostock.de \\ 4 Animal Breeding and Genetics, Faculty of Agricultural and Environmental Sciences, University of Rostock, \\ 18059 Rostock, Germany \\ * Correspondence: wimmers@fbn-dummerstorf.de; Tel.: +49-38208-68-600
}

check for updates

Citation: Reyer, H.; Sjöberg, P.J.R.; Oster, M.; Wubuli, A.; Murani, E.; Ponsuksili, S.; Wolf, P.; Wimmers, K. Mineral Phosphorus Supply in Piglets Impacts the Microbial Composition and Phytate Utilization in the Large Intestine. Microorganisms 2021, 9, 1197. https://doi.org/10.3390/

microorganisms 9061197

Academic Editor: Ryo Inoue

Received: 7 May 2021

Accepted: 28 May 2021

Published: 1 June 2021

Publisher's Note: MDPI stays neutral with regard to jurisdictional claims in published maps and institutional affiliations.

Copyright: (c) 2021 by the authors. Licensee MDPI, Basel, Switzerland. This article is an open access article distributed under the terms and conditions of the Creative Commons Attribution (CC BY) license (https:// creativecommons.org/licenses/by/ $4.0 /)$.

\begin{abstract}
A sufficient supply of phosphorus (P) to pigs in livestock farming is based on the optimal use of plant-based phytate and mineral $\mathrm{P}$ supplements to ensure proper growth processes and bone stability. However, a high P supplementation might bear the risk of higher environmental burden due to the occurrence of excess $\mathrm{P}$ and phytate degradation products in manure. In this context, the intestinal microbiota is of central importance to increase P solubility, to employ non-mineral $\mathrm{P}$ by the enzymatic degradation of phytate, and to metabolize residual P. A feeding experiment was conducted in which piglets were fed diets with different $\mathrm{P}$ levels, resulting in three groups with low, medium (covering requirements), and high concentrations of available P. Samples from caecum and colon digesta were analysed for microbial composition and phytate breakdown to estimate the microbial contribution to metabolize P sources. In terms of identified operational taxonomic units (OTU), caecum and colon digesta under the three feeding schemes mainly overlaP in their core microbiome. Nevertheless, different microbial families correlate with increased dietary P supply. Specifically, microbes of Desulfovibrionaceae, Pasteurellaceae, Anaerovoracaceae, and Methanobacteriaceae were found significantly differentially abundant in the large intestine across the dietary treatments. Moreover, members of the families Veillonellaceae, Selenomonadaceae, and Succinivibrionaceae might contribute to the observed phytate degradation in animals fed a low P diet. In this sense, the targeted manipulation of the intestinal microbiota by feeding measures offers possibilities for the optimization of intestinal phytate and P utilization.
\end{abstract}

Keywords: phosphorus; inositolphosphate; large intestine; 16S rRNA; pigs

\section{Introduction}

The viability of all organisms depends on a demand-covering supply of phosphorus (P) for the establishment of structural body compartments and the maintenance of other important physiological functions, such as energy supply, cell signalling, and blood buffering. For pigs, the main $\mathrm{P}$ sources are phosphorus-containing mineral supplementations (dicalcium-/monocalcium-phosphates) produced from rock phosphates and also phytic acid (myo-inositol 1,2,3,4,5,6-hexakis; InsP6), which is usually present as phytate and represents the storage form of $\mathrm{P}$ in plants [1,2]. The provision of $\mathrm{P}$ available to the animal from phytate is essential to meet the needs of the organism while using a sustainable mineral source. However, the phytate utilization requires its gradual hydrolysis, which is catalysed by phytases and other phosphatases, such as the intestinal alkaline phosphatase. 
Depending on dietary supply and gastrointestinal site, the cleavage steps result in variable amounts of phosphate, inositolphosphates (InsPx), and myo-inositol. However, due to the low impact of endogenous intestinal phosphatases on phytate hydrolysis, pigs have a low capacity to utilise phytic acid, which requires the involvement of phytases of plant or microbial origin [3]. In practice, exogenous phytases of microbial origin are added to pig feed, improving the available $\mathrm{P}$ content based on the amount and origin of phytases [2]. However, considering the microbial community of the gastrointestinal tract of pigs and chickens, it is known that some of the microbes present are able to produce phytase and secrete them into the lumen. These include Bifidobacteria [4], various isolates of Lactobacillus [5,6], and Pediococcus [7] as well as Pseudomonas spp. [8]. This exemplifies the potentially broad microbial capacity to degrade phytate and provide $\mathrm{P}$ in the gastrointestinal tract of monogastric animal species. Besides the benefits of phytate as a P source, antinutritive effects of InsP6 (inositol hexakisphosphate), InsP5 (inositol pentakisphosphate), InsP4 (inositol tetrakisphosphate), and InsP3 (inositol triphosphate) have been described. These include mainly the impairment of the protein-degradation capacity of pepsin and the drastic reduction of the solubility of zinc at $\mathrm{pH}$ values relevant for the proximal small intestine in the presence of InsPx [2,9]. Therefore, an efficient breakdown of phytate in the gastrointestinal tract is preferable. Moreover, there is an interaction of dietary mineral P and InsPx such that high $P$ hampers the degradation of phytase [10]. At present, several approaches proved beneficial for the rapid hydrolysis of phytate, e.g., liquid feeding, phytase superdosing, or feed pre-treatment $[11,12]$. Considering the above-mentioned potential of microbes present in the gastrointestinal tract to provide phytases and improve P efficiency, dietary strategies might further include the establishment of a favourable intestinal microbiome profile. Shifts in the microbial composition of the intestine will facilitate meeting the required P supply by exploiting a higher degree of non-mineral $\mathrm{P}$ of plant origin to reduce $\mathrm{P}$ excretion and thus to reduce the environmental impact. Therefore, the current study focuses on the investigation of the microbial composition and the profile of inositolphophates in the pig large intestine in response to varying dietary $\mathrm{P}$ intakes.

\section{Materials and Methods}

\subsection{Animal Trial and Sample Preparation}

The animal experiment to which this study refers was approved by the ethics committee of the federal state of Mecklenburg-Western Pomerania, Germany (Landesamt für Landwirtschaft, Lebensmittelsicherheit und Fischerei; LALLF-M-V/TSD/7221.3-1-053-15). A feeding trial was carried out in an experimental pig house with varying levels of mineral $\mathrm{P}$ fed to piglets between 28 and 64 days of life, as previously described [13]. In brief, dietary groups were composed as follows: grouP L consisting of 8 piglets fed with a lower mineral $\mathrm{P}$ level $(\mathrm{P}=0.6 \%$ of $\mathrm{DM})$, grouP $\mathrm{M}$ consisting of 6 piglets fed recommended $\mathrm{P}$ levels $(\mathrm{P}=0.9 \%$ of $\mathrm{DM})$, and grouP $\mathrm{H}$ consisting of 7 piglets fed with a higher mineral $\mathrm{P}$ level $(\mathrm{P}=1.1 \%$ of $\mathrm{DM})$. Except $\mathrm{P}$, all other nutrient contents of the feed were based on current recommendations [14]. The diets had comparable levels of protein (L: 205 g/kg DM; M: 203 g/kg DM; H: 201 g/kg DM), metabolizable energy (L: $12.5 \mathrm{MJ} / \mathrm{kg}$ DM; M: $12.5 \mathrm{MJ} / \mathrm{kg}$ DM; H: $12.3 \mathrm{MJ} / \mathrm{kg} \mathrm{DM}$ ), and calcium (L: $13.0 \mathrm{~g} / \mathrm{kg} \mathrm{DM;}$ M: $13.0 \mathrm{~g} / \mathrm{kg}$ DM; H: $13.0 \mathrm{~g} / \mathrm{kg}$ DM) [13]. Vitamin D3 was provided at the level of $1000 \mathrm{IU}$ for all dietary groups. No microbial phytases were added to the wheat/barley/soybean meal-based diets. The piglets originated from 4 litters and were equally assigned to dietary groups with at least 3 males and females per group. The trial was carried out in March-April at ambient stable temperature and relative humidity. A constant 12-h lightdark cycle was applied. The piglets were kept individually on a flat-deck and had ad libitum access to pelleted feed and water [13]. At the end of the trial period, pigs were killed by exsanguination following electric stunning. From all pigs, digesta samples were collected from the terminal tiP of the caecum and the mid region of the colon. Subsequently, samples were shock frozen in liquid nitrogen and stored at $-80{ }^{\circ} \mathrm{C}$ until microbiota and inositolphosphate analyses. 


\section{2. $16 S$ rRNA Profiling}

DNA extraction of pig digesta samples followed the instructions of the PowerLyzer PowerSoil DNA Isolation Kit (MoBio, Carlsbad, CA, USA) with additional heating steps of $10 \mathrm{~min}$ at $70^{\circ} \mathrm{C}$ and $95^{\circ} \mathrm{C}$ prior to beat beating. Amplificates of the $16 \mathrm{~S}$ rRNA gene were produced in duplicates using primers specific for variable region $\mathrm{V} 4\left(515^{\prime} \mathrm{F}\right.$ and $\left.806 \mathrm{R}\right)$, including adapters and barcodes $[15,16]$. Polymerase chain reactions were performed with 5PRIME HotMasterMix (5 PRIME, Hamburg, Germany) as follow: $95^{\circ} \mathrm{C}$ for $2 \mathrm{~min}$, 30 cycles at $95^{\circ} \mathrm{C}$ for $30 \mathrm{~s}, 55^{\circ} \mathrm{C}$ for $60 \mathrm{~s}, 72^{\circ} \mathrm{C}$ for $90 \mathrm{~s}$, and a final extension for $10 \mathrm{~min}$ at $72{ }^{\circ} \mathrm{C}$. PCR products were purified using magnetic beads (Agencourt AMPure XP, Beckman Coulter, Krefeld, Germany) and mixed in equal concentrations. Sequencing was performed on a HiSeq2500 (Illumina, San Diego, CA, USA) generating 250 bP paired-end reads. After demultiplexing of the sequencing reads, raw data were analysed with the mothur software (version 1.44.1) [17]. Sequences were globally aligned to the Silva reference database (release 138) with chimeric sequences removed. Considering a sequence identity of $97 \%$, the sequences were combined into operational taxonomic units (OTU), and OTU annotations were retrieved from the Silva database (release 138).

\subsection{Inositolphosphate Analysis}

For the analysis of InsP6 and InsP5, approximately $200 \mathrm{mg}$ of digesta samples obtained from caecum and colon were lyophilized. The chemicals used were of analytical quality (Sigma-Aldrich, Taufkirchen, Germany). In-house stock solution was prepared as previously described $[18,19]$ from the dipotassium salt of myo-inositol hexakis (dihydrogenphosphate) (P5681, Sigma-Aldrich, Taufkirchen, Germany). A standard series was prepared by diluting the in-house stock solution with $0.1 \mathrm{M} \mathrm{NaOH}$ containing $0.01 \mathrm{M}$ etylenediamin tetraacetic acid (EDTA) in the range between $0.1-55 \mu \mathrm{M}$ for calibration of the ESI-MS system. Freeze-dried samples (approx. $20 \mathrm{mg}$ ) were extracted with $1 \mathrm{~mL} 1.0 \mathrm{M}$ $\mathrm{NaOH}$ containing $0.1 \mathrm{M}$ EDTA. Samples were shaken with a Multi Reax for $16 \mathrm{~h}$ (Heidolph Instrument, Schwabach, Germany). To remove particles, the samples were centrifuged at $10,000 \mathrm{rpm}$ for $15 \mathrm{~min}$. The supernatant $(0.1 \mathrm{~mL})$ was mixed with $0.9 \mathrm{~mL}$ of Milli-Q water in a glass vial before placing it in the LC autosampler for analysis. The 1260 Infinity (Agilent Technologies, Waldbronn, Germany) chromatographic system was used in connection with a 3200 Q TraP LC/MS/MS system (AB Sciex, Concord, ON, Canada). The LC-MS method was the same as previously reported [19]. InsP6 and InsP5 were detected by using multiple reaction monitoring mode (MRM) with suitable precursor and product ion transitions with optimal collision energy (CE) and collision cell exit potential (CXP). These values were evaluated in a previous work [19]. Data acquisition and quantification were carried out with Analyst 1.4.2 (AB Sciex). All MRM transitions for respective InsPx were summed before manual peak integration.

\subsection{Data Analysis}

The OTU abundances determined were adjusted by means of subsampling, taking into account the library with the fewest sequences. After taxonomic annotation of OTU, the relative abundance of phyla and family level were visualized in taxa plots employing the $R$ software. Dietary differences at family level were assessed separately for caecum and colon using DESeq2 (DOI:10.18129/B9.bioc.DESeq2). Therefore, very low abundant families were excluded by considering only taxa with more than ten observations in at least $30 \%$ of all samples. A likelihood ratio test was performed against a base model with mother and sex of piglets as effects. Differences were considered significant at a Benjamini-Hochberg adjusted $p$-value $<0.05$. Data referring to the InsPx levels in caecum and colon contents were summarized as mean $\pm \mathrm{SE}$. One sample from the $\mathrm{L}$ grouP had to be removed from the analyses due to missing values. Data were analysed in a linear model ( $R$ package lmerTest, v3.1-2) considering effects of dietary P content, litter, and sex. The significance threshold was set at $p<0.05$. For correlation analysis between phenotypes and microbiota, Kendall correlation coefficients were calculated. Therefore, animal-individual values of 
P intake and serum P levels were obtained from a previous study and considered in the analysis [13].

\section{Results and Discussion}

The measurement of inositolphosphates in caecum and colon digesta revealed a gradual increase of InsP6 and InsP5 in the two intestinal sections with increasing mineral P supply in the diets (Figure 1). The L grouP showed significantly lower levels of InsP6 and InsP5 in both intestinal segments compared to $\mathrm{M}$ and $\mathrm{H}(p<0.05)$, although this difference was not considered significant when comparing InsP5 levels between L and M ( $p=0.05$ for colon, $p=0.08$ for caecum). Despite the absence of a phytase of exogenous origin in the feed, there is a clear response to reduced mineral $\mathrm{P}$ supply in the $\mathrm{L}$ grouP resulting in an increased hydrolysis of phytate. Supporting this observation, it was found that phytase activity in the ileum increased with decreasing $\mathrm{P}$ in the diet [20]. The group-specific patterns and magnitude of effects were similar for caecum and colon digesta, although, a higher amount of inositolphosphates was found for $\mathrm{H}$ in the colon compared to the caecum $(p<0.05$ for InsP6 and InsP5).
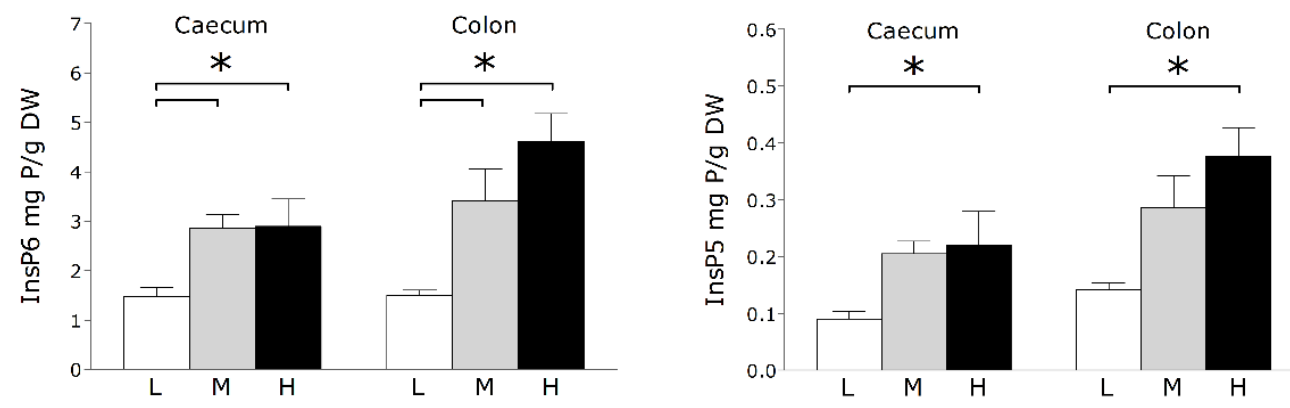

Figure 1. Levels of inositol hexakisphosphate (InsP6) and inositol pentakisphosphate (InsP5) in the caecum and colon of piglets fed a varying supply of mineral $\mathrm{P}$ for a period of 5 weeks. Significant differences are indicated by an asterisk ( $\left.{ }^{*} ; p<0.05\right)$. L, M, H-dietary groups receiving lower, medium, and higher mineral P levels; DW—dry weight.

Since the experimental design excluded exogenous phytase supplements, the main factor for the observed differences in an inositolphosphate degradation likely refers to the intestinal microbiota composition. The sequencing of the $16 \mathrm{~S}$ rRNA revealed that, at OTU level, about two-thirds of the taxonomic units overlapped between caecum and colon digesta (Figure 2A). This corresponds to the previous observation that caecum and colon exhibit a high similarity in microbiota profiles [21]. Regarding the specific dietary groups in both tissues, the relations represented in the Venn diagrams revealed a considerable amount of common OTU interpreted as independent of the dietary P supply (Figure 2B,C). However, 39 to 60 OTUs were identified to be specific for a single dietary group, without a particular grouP displaying a considerable increase in microbial richness. Interestingly, the overlaP in OTUs was numerically higher in the extreme P groups (239 and 186 in caecum and colon, respectively, considering $\mathrm{L}$ and $\mathrm{H}$ ) compared to the contrasts with the $\mathrm{M}$ group. This might be related to observations that varying dietary $\mathrm{P}$ content and phytase addition are associated with the capacity of the cellular adaptive immune system and influence the metabolism in terms of fibre digestion and the concentration of microbial metabolites [22-24]. 
A

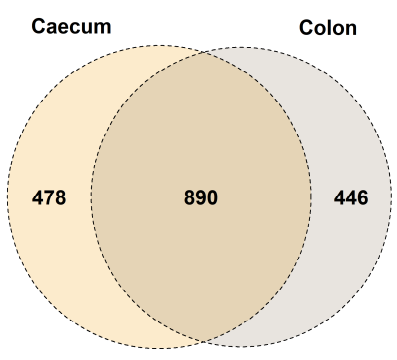

B

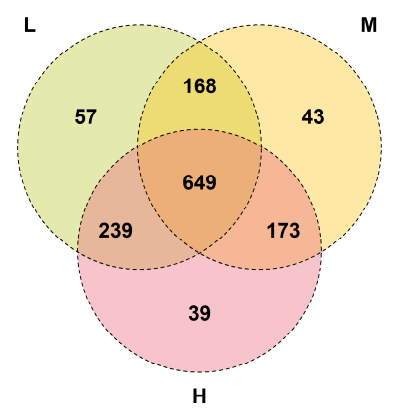

C

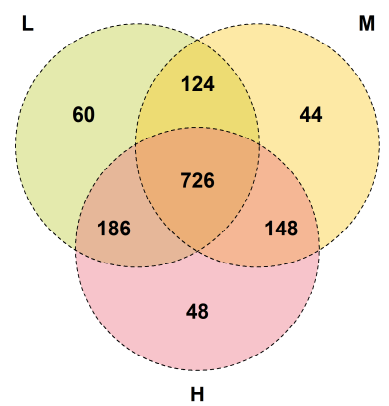

D

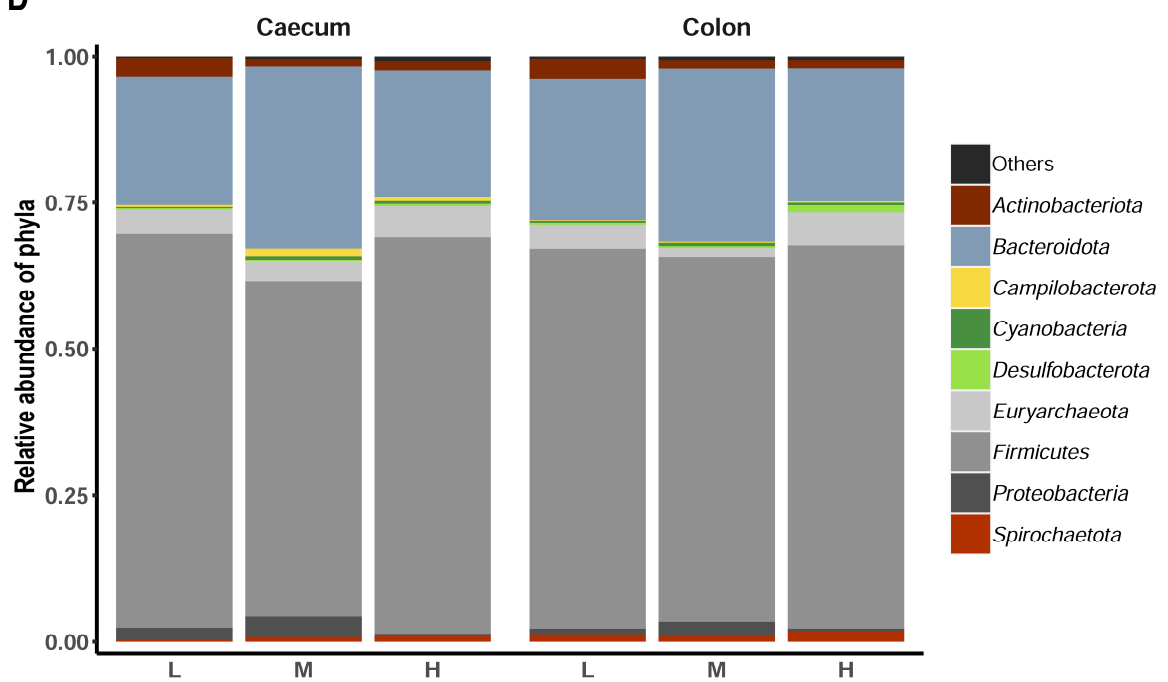

E

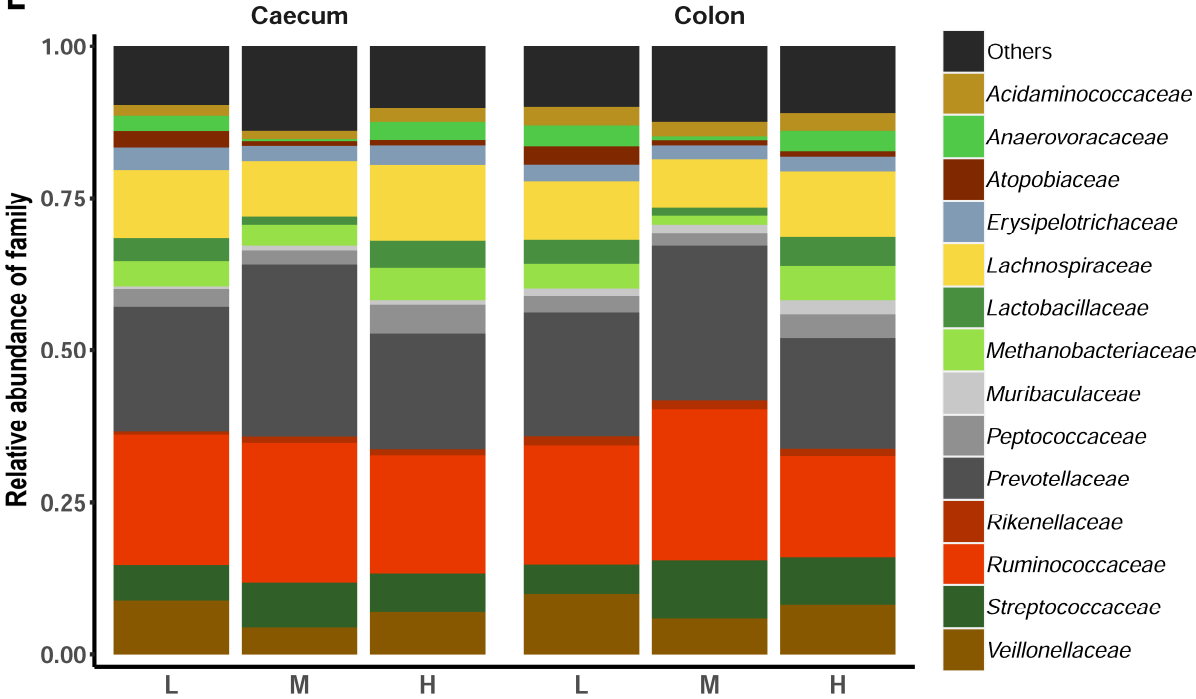

Figure 2. Microbial composition of caecum and colon digesta of pigs fed with varying mineral P levels. Venn diagram representation of specific and overlapping OTUs considering (A) caecum and colon digesta samples, (B) caecum digesta samples from the three dietary groups, and (C) colon digesta samples from the dietary groups. Taxa plots at (D) phylum and (E) family level with the 9 and 14 toP taxa, respectively. L, M, H-dietary groups receiving lower, medium, and higher mineral P levels.

Diet-specific taxa plots at phylum level revealed a large overlaP in the microbiota composition between the two intestinal segments and the three dietary groups (Figure 2D). The most abundant phyla comprised Firmicutes and Bacteroidota, with an average relative abundance of 0.64 and 0.25 , respectively. The abundance of these two predominant phyla reflects the typical microbiota composition in the pig at this age [21]. Group-specific differences in the average abundance were consistent across tissue sites. Considering the toP 14 taxa at the family level, Prevotellaceae and Ruminococcaceae dominated in caecum and colon (Figure 2E). Statistical analysis at family level revealed that microbes assigned to Anaerovoracaceae and Pasteurellaceae in the caecal digesta were found to be significantly differentially abundant between dietary groups (adjusted $p<0.05$, Figure 3, Supplementary Tables S1 and S2). As shown in the animal-individual representation of abundances of Pasteurellaceae in Figure 3A, the differences were mainly driven by two samples of the $\mathrm{M}$ grouP showing a considerably high proportion of sequences assigned to this taxon. For Anaerovoracaceae, samples of $\mathrm{L}$ and $\mathrm{H}$ groups showed higher abundance compared to $\mathrm{M}$ 
animals. In the colon digesta, Anaerovoracaceae were also differentially abundant between the dietary groups on family classification, showing the same diet-specific pattern likewise in caecum. The family Anaerovoracaceae is sparsely characterized. It belongs to the class of Clostridia, which are typically involved in the fermentation of plant polysaccharides in the gastrointestinal tract [25]. In colon digesta, also Methanobacteriaceae and Desulfovibrionaceae differed significantly in their abundance between dietary groups. Methanobacteriaceae was lowest abundant in $\mathrm{M}$, whereas microbes of Desulfovibrionaceae were found predominantly in the colon digesta of $\mathrm{H}$ animals. The predominant process for energy generation in Methanobacteriaceae is the reduction of carbon dioxide to form methane [26]. However, it has recently been shown that the abundance of Archaeae, which include Methanobacteriaceae, is correlated to phytase supplementation in pigs [27]. For Desulfovibrionaceae, the genus Desulfovibrio was found to have a significantly higher relative abundance in the jejunum and caecum of goats with a high digestibility of $\mathrm{P}$ [28]. Therefore, independent of the actual functional involvement in P metabolism, representative species of these two families could drive potential improvements of $P$ availability and phytate utilization in the gastrointestinal tract of pigs.
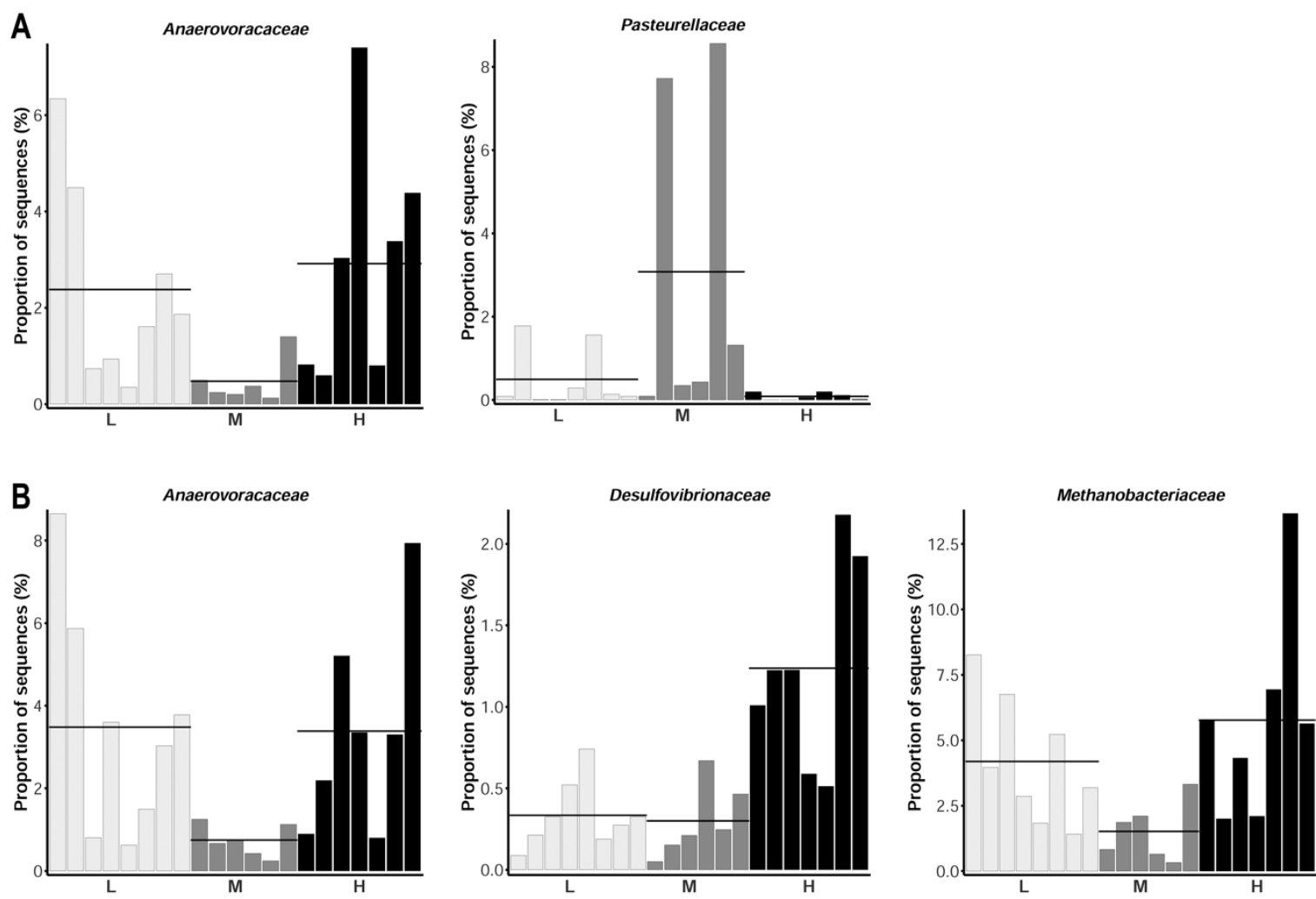

Figure 3. Significant differentially abundant taxa in (A) caecum and (B) colon digesta at family level (adjusted $p<0.05$ ). Individual proportions of sequences assigned to the taxa are represented for each of the three dietary groups. L, M, $\mathrm{H}-$ dietary groups receiving lower, medium, and higher mineral P supplements.

The variable dietary P supply provided to the pigs in this study affects various levels of the organism such as the chemical composition and the microstructure of the bones as well as the hormone level and the mineral concentration in the blood to primarily maintain $\mathrm{P}$ homeostasis [13]. In order to further explore the interaction between dietary P intake, inositolphosphate degradation, and the abundance of microbial taxa in the caecum and colon, correlation analyses were performed. The majority of the 25 most abundant families included in the analysis originated from Firmicutes (Figure 4). With respect to the microbial families with higher relative abundance, significant positive correlation coefficients were identified for Streptococcaceae with P intake und serum P levels in colon and with InsP6 
values in caecum. This implies favourable conditions for the growth of Streptococcaceae under higher available P concentrations in the digesta. It has been described that species of Streptococcaceae perform proteolytic processes in the colon and contribute substantially to the turnover of sulfur-containing substrates [29]. Phytase activity has not yet been reported for representatives of this microbial family. Veillonellaceae were significantly negatively correlated with InsP6 levels in caecum digesta. This indicates an increased phytate utilization in the presence of microbes of this family. Interestingly, by adding exogenous phytase to the diets of growing pigs, the relative abundance of Veillonellaceae significantly decreased [27]. For Lactobacillaceae, a positive correlation with InsP6 and InsP5 in colon was retrieved. For low abundant taxa, increasing levels of dietary mineral $P$ intake were suggested to correlate with an increased presence of Muribaculaceae, Rikenellaceae, and Desulfovibrionaceae. For Muribaculaceae and Rikenellaceae, so far, no information is available about their involvement in P metabolism. Members of the former are known for degradation of carbohydrates and utilization of nitrogen, whereas the latter are mainly hydrogen-producing bacteria [30]. In accordance with the results of the statistical comparison of dietary groups in this study, the identification of Desulfovibrionaceae provides clear evidence of a relationshiP between the dietary intake of mineral $\mathrm{P}$ and the occurrence of members of this microbial family. The abundance of Oscillospiraceae was significantly positively correlated with P intake, InsP6, and InsP5 abundance and serum P levels in both intestinal segments. These conditions of higher dietary $\mathrm{P}$ availability and a higher phytate reservoir could lead to conditions with more available $P$ in the large intestine, which represent favourable conditions for certain microbes. Indeed, members of the Oscillospiraceae were previously shown to be increased abundantly due to phytase supplementation [27]. Moreover, other representatives of the order Oscillospirales, namely Oscillospirales_fa were positively correlated with caecum InsP6 and InsP5 as well as colon InsP5. Selenomonadaceae and Succinivibrionaceae showed significant negative correlation coefficients with InsP6 and InsP5 levels in different parts of the large intestine. Further, the abundance of Selenomonadaceae in the colon was significantly correlated with P intake and serum P levels. Interestingly, a certain phytase activity was detected in several species belonging to the Selenomonadaceae, among which, e.g., Mitsuokella were considered to have a high activity [31].

Besides the essential capacity for P absorption in the small intestine, the role of $\mathrm{P}$ metabolism in the caecum and colon is still largely unclear. It is known for certain that $\mathrm{P}$ is released from phytic acid by hydrolysis in the large intestine of pigs, which in turn is available for use by microbial species [32]. However, there are various indications for an uptake of $\mathrm{P}$ into the organism also via the epithelium of the large intestine [23]. Specifically, indications are provided by the post-ileal disappearance of $\mathrm{P}$ and the expression of P-transporters in the terminal colon $[33,34]$. 


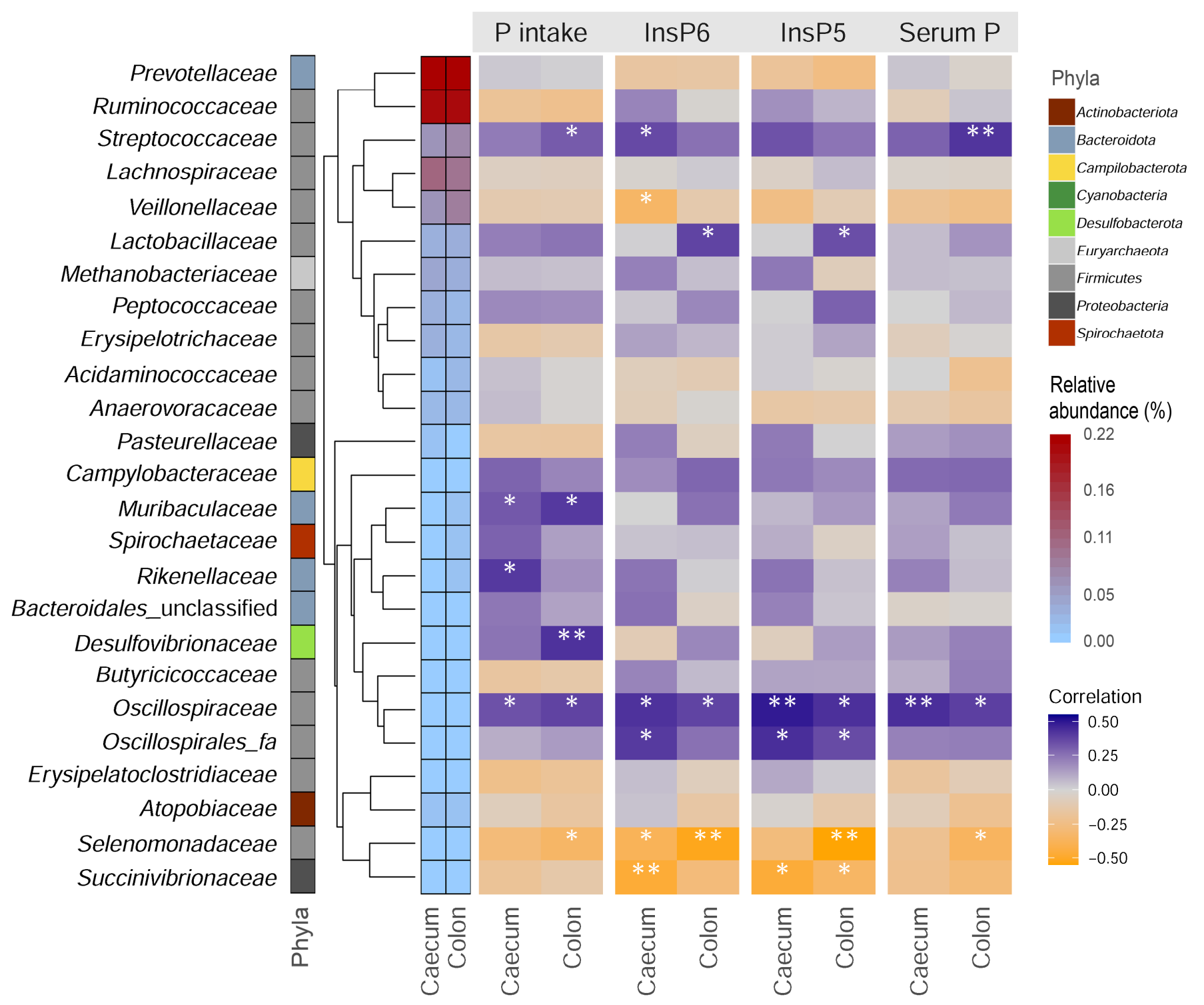

Figure 4. Correlation heatmap. Data of the 25 most abundant microbial families detected in the large intestine were correlated with phosphorus (P) intake, inositol-6 phosphate (InsP6), inositol-5 phosphate (InsP5), and serum P levels. The phyla column indicates the assignment of families to phyla. The cladogram represents the hierarchical clustering of taxa based on their abundance. Correlation coefficients considered significant are indicated by an asterisk $\left(*: p<0.05,{ }^{* *}: p<0.01\right)$.

\section{Conclusions}

The current study identifies a number of microbial taxa that either influence the $P$ availability in the large intestine through their phytase activity or are positively or negatively influenced by higher intestinal P levels in terms of growth and replication. Microbial families that might benefit from increasing dietary P supply comprise Streptococcaceae, Muribaculaceae, Rikenellaceae, Desulfovibrionaceae, and Oscillospiraceae. Potential to provide additional phytase activity and thus induce the hydrolyses of inositolphosphates might be contributed by members of the families Veillonellaceae, Selenomonadaceae, and Succinivibrionaceae. A P-reduced diet for pigs triggers microbial-mediated compensatory actions through phytate cleavage and $\mathrm{P}$ release, thereby increasing available $\mathrm{P}$ to the host and gut microbiota. In addition to changes in the abundance of certain taxa associated with $\mathrm{P}$ digestion and inositol degradation, changes in microbial enzyme expression and 
functional shifts of microbes in response to P-variable diets may also be important and can be revealed by metatranscriptome analysis.

Supplementary Materials: The following are available online at https:/ / www.mdpi.com/article/10 .3390 / microorganisms9061197/s1, Table S1: Group-specific proportions of sequences assigned to taxa at family level in the caecum digesta; Table S2: Group-specific proportions of sequences assigned to taxa at family level in the colon digesta.

Author Contributions: Conceptualization, K.W. and P.W.; methodology, A.W., H.R., M.O., P.J.R.S., and S.P.; formal analysis, H.R., M.O., and K.W.; investigation, M.O., K.W., and P.W.; resources, E.M., P.J.R.S., S.P., and K.W.; data curation, H.R. and M.O.; writing-original draft preparation, H.R.; writing-review and editing, A.W., M.O., P.J.R.S., E.M., S.P., P.W., and K.W.; visualization, H.R.; supervision, K.W.; funding acquisition, P.W. and K.W. All authors have read and agreed to the published version of the manuscript.

Funding: This study has received funding from the European Research Area Network (ERA-NET) co-funds on Sustainable Animal Production (SusAn) as part of the PEGaSus project (2817ERA02D). This work was partly funded by the Leibniz ScienceCampus Phosphorus Research Rostock.

Institutional Review Board Statement: The study was conducted according to the guidelines of the Declaration of Helsinki, approved by the Animal Welfare Committee of the FBN, and was licensed by the Ethics Committee of the federal state of Mecklenburg-Western Pomerania, Germany (LALLF-M-V/TSD/7221.3-1-053-15; date of approval: 16 December 2015).

Informed Consent Statement: Not applicable.

Acknowledgments: The authors thank Angela Garve and Frieder Hadlich for their excellent technical help.

Conflicts of Interest: The authors declare no conflict of interest.

\section{References}

1. Oster, M.; Reyer, H.; Ball, E.; Fornara, D.; McKillen, J.; Sørensen, K.U.; Poulsen, H.D.; Andersson, K.; Ddiba, D.; Rosemarin, A. Bridging gaps in the agricultural phosphorus cycle from an animal husbandry perspective-The case of pigs and poultry. Sustainability 2018, 10, 1825. [CrossRef]

2. Humer, E.; Schwarz, C.; Schedle, K. Phytate in pig and poultry nutrition. J. Anim. Physiol. Anim. Nutr. 2015, 99, 605-625. [CrossRef]

3. Schlemmer, U.; Jany, K.D.; Berk, A.; Schulz, E.; Rechkemmer, G. Degradation of phytate in the gut of pigs-pathway of gastrointestinal inositol phosphate hydrolysis and enzymes involved. Arch. Anim. Nutr. 2001, 55, 255-280. [CrossRef]

4. Haros, M.; Bielecka, M.; Sanz, Y. Phytase activity as a novel metabolic feature in bifidobacterium. FEMS Microbiol. Lett. 2005, 247, 231-239. [CrossRef] [PubMed]

5. Kim, E.-Y.; Kim, Y.-H.; Rhee, M.-H.; Song, J.-C.; Lee, K.-W.; Kim, K.-S.; Lee, S.-P.; Lee, I.-S.; Park, S.-C. Selection of Lactobacillus sp. Psc101 that produces active dietary enzymes such as amylase, lipase, phytase and protease in pigs. J. Gen. Appl. Microbiol. 2007, 53, 111-117. [CrossRef]

6. Asghar, S.; Arif, M.; Nawaz, M.; Muhammad, K.; Ali, M.; Ahmad, M.; Iqbal, S.; Anjum, A.; Khan, M.; Nazir, J. Selection, characterisation and evaluation of potential probiotic lactobacillus spp. isolated from poultry droppings. Benef. Microbes 2016, 7, 35-44. [CrossRef]

7. Raghavendra, P.; Halami, P.M. Screening, selection and characterization of phytic acid degrading lactic acid bacteria from chicken intestine. Int. J. Food Microbiol. 2009, 133, 129-134. [CrossRef]

8. Hosseinkhani, B.; Hosseinkhani, G. Analysis of phytase producing bacteria (Pseudomonas sp.) from poultry faeces and optimization of this enzyme production. Afr. J. Biotechnol 2009, 8, 4229-4232.

9. Yu, S.; Cowieson, A.; Gilbert, C.; Plumstead, P.; Dalsgaard, S. Interactions of phytate and myo-inositol phosphate esters (IP1-5) including IP5 isomers with dietary protein and iron and inhibition of pepsin. J. Anim. Sci. 2012, 90, 1824-1832. [CrossRef] [PubMed]

10. Shastak, Y.; Zeller, E.; Witzig, M.; Schollenberger, M.; Rodehutscord, M. Effects of the composition of the basal diet on the evaluation of mineral phosphorus sources and interactions with phytate hydrolysis in broilers. Poult. Sci. 2014, 93, 2548-2559. [CrossRef] [PubMed]

11. Blaabjerg, K.; Jørgensen, H.; Tauson, A.-H.; Poulsen, H.D. Heat-treatment, phytase and fermented liquid feeding affect the presence of inositol phosphates in ileal digesta and phosphorus digestibility in pigs fed a wheat and barley diet. Animal 2010, 4, 876-885. [CrossRef] [PubMed] 
12. Lu, H.; Cowieson, A.J.; Wilson, J.W.; Ajuwon, K.M.; Adeola, O. Extra-phosphoric effects of super dosing phytase on growth performance of pigs is not solely due to release of myo-inositol. J. Anim. Sci. 2019, 97, 3898-3906. [CrossRef] [PubMed]

13. Gerlinger, C.; Oster, M.; Reyer, H.; Polley, C.; Vollmar, B.; Muráni, E.; Wimmers, K.; Wolf, P. Effects of excessive or restricted phosphorus and calcium intake during early life on markers of bone architecture and composition in pigs. J. Anim. Physiol. Anim. Nutr. 2020. [CrossRef]

14. Gesellschaft Für Ernährungsphysiologie (GFE). Empfehlungen zur Energie-und Nährstoffversorgung beim Schwein. In Ausschuss für Bedarfsnormen der Gesellschaft für Ernährungsphysiologie; DLG Verlag: Frankfurt, Germany, 2006.

15. Kozich, J.J.; Westcott, S.L.; Baxter, N.T.; Highlander, S.K.; Schloss, P.D. Development of a dual-index sequencing strategy and curation pipeline for analyzing amplicon sequence data on the MiSeq Illumina sequencing platform. Appl. Environ. Microbiol. 2013, 79, 5112-5120. [CrossRef] [PubMed]

16. Hugerth, L.W.; Wefer, H.A.; Lundin, S.; Jakobsson, H.E.; Lindberg, M.; Rodin, S.; Engstrand, L.; Andersson, A.F. Degeprime, a program for degenerate primer design for broad-taxonomic-range pcr in microbial ecology studies. Appl. Environ. Microbiol. 2014, 80, 5116-5123. [CrossRef] [PubMed]

17. Schloss, P.D.; Westcott, S.L.; Ryabin, T.; Hall, J.R.; Hartmann, M.; Hollister, E.B.; Lesniewski, R.A.; Oakley, B.B.; Parks, D.H.; Robinson, C.J. Introducing mothur: Open-source, platform-independent, community-supported software for describing and comparing microbial communities. Appl. Environ. Microbiol. 2009, 75, 7537-7541. [CrossRef] [PubMed]

18. Paraskova, J.V.; Jørgensen, C.; Reitzel, K.; Pettersson, J.; Rydin, E.; Sjöberg, P.J. Speciation of inositol phosphates in lake sediments by ion-exchange chromatography coupled with mass spectrometry, inductively coupled plasma atomic emission spectroscopy, and 31P NMR spectroscopy. Anal. Chem. 2015, 87, 2672-2677. [CrossRef]

19. Sjöberg, P.J.; Thelin, P.; Rydin, E. Separation of inositol phosphate isomers in environmental samples by ion-exchange chromatography coupled with electrospray ionization tandem mass spectrometry. Talanta 2016, 161, 392-397. [CrossRef]

20. Seynaeve, M.; Janssens, G.; Hesta, M.; Van Nevel, C.; De Wilde, R. Effects of dietary Ca/P ratio, P level and microbial phytase supplementation on nutrient digestibilities in growing pigs: Breakdown of phytic acid, partition of $\mathrm{P}$ and phytase activity along the intestinal tract. J. Anim. Physiol. Anim. Nutr. 2000, 83, 193-204. [CrossRef]

21. De Rodas, B.; Youmans, B.P.; Danzeisen, J.L.; Tran, H.; Johnson, T.J. Microbiome profiling of commercial pigs from farrow to finish. J. Anim. Sci. 2018, 96, 1778-1794. [CrossRef]

22. Metzler-Zebeli, B.U.; Mann, E.; Schmitz-Esser, S.; Wagner, M.; Ritzmann, M.; Zebeli, Q. Changing dietary calcium-phosphorus level and cereal source selectively alters abundance of bacteria and metabolites in the upper gastrointestinal tracts of weaned pigs. Appl. Environ. Microbiol. 2013, 79, 7264-7272. [CrossRef]

23. Heyer, C.M.; Weiss, E.; Schmucker, S.; Rodehutscord, M.; Hoelzle, L.E.; Mosenthin, R.; Stefanski, V. The impact of phosphorus on the immune system and the intestinal microbiota with special focus on the pig. Nutr. Res. Rev. 2015, 28, 67-82. [CrossRef] [PubMed]

24. Heyer, C.M.E.; Schmucker, S.; Burbach, K.; Weiss, E.; Eklund, M.; Aumiller, T.; Capezzone, F.; Steuber, J.; Rodehutscord, M.; Hoelzle, L.E. Phytate degradation, intestinal microbiota, microbial metabolites and immune values are changed in growing pigs fed diets with varying calcium-phosphorus concentration and fermentable substrates. J. Anim. Physiol. Anim. Nutr. 2019, 103, 1185-1197. [CrossRef] [PubMed]

25. Boutard, M.; Cerisy, T.; Nogue, P.-Y.; Alberti, A.; Weissenbach, J.; Salanoubat, M.; Tolonen, A.C. Functional diversity of carbohydrate-active enzymes enabling a bacterium to ferment plant biomass. PLoS Genet. 2014, 10, e1004773. [CrossRef] [PubMed]

26. Hedderich, R.; Whitman, W.B. Physiology and Biochemistry of the Methane-Producing Archaea. In The Prokaryotes: Volume 2: Ecophysiology and Biochemistry; Dworkin, M., Falkow, S., Rosenberg, E., Schleifer, K.-H., Stackebrandt, E., Eds.; Springer: New York, NY, USA, 2006; pp. 1050-1079.

27. Klinsoda, J.; Vötterl, J.; Koger, S.; Metzler-Zebeli, B.U. Dietary phytase and lactic acid treated cereals caused greater taxonomic than functional adaptations in the cecal metagenome of growing pigs. Appl. Environ. Microbiol. 2020, 87, e02240-20. [CrossRef] [PubMed]

28. Wang, L.; Shah, A.M.; Liu, Y.; Jin, L.; Wang, Z.; Xue, B.; Peng, Q. RelationshiP between true digestibility of dietary phosphorus and gastrointestinal bacteria of goats. PLOS ONE 2020, 15, e0225018. [CrossRef]

29. Pieper, R.; Tudela, C.V.; Taciak, M.; Bindelle, J.; Pérez, J.F.; Zentek, J. Health relevance of intestinal protein fermentation in young pigs. Anim. Health Res. Rev. 2016, 17, 137-147. [CrossRef]

30. Lagkouvardos, I.; Lesker, T.R.; Hitch, T.C.; Gálvez, E.J.; Smit, N.; Neuhaus, K.; Wang, J.; Baines, J.F.; Abt, B.; Stecher, B. Sequence and cultivation study of Muribaculaceae reveals novel species, host preference, and functional potential of this yet undescribed family. Microbiome 2019, 7, 1-15. [CrossRef]

31. Yanke, L.; Bae, H.; Selinger, L.; Cheng, K. Phytase activity of anaerobic ruminal bacteria. Microbiology 1998, 144, 1565-1573. [CrossRef]

32. Leytem, A.B.; Turner, B.L.; Thacker, P. Phosphorus composition of manure from swine fed low-phytate grains: Evidence for hydrolysis in the animal. J. Environ. Qual. 2004, 33, 2380-2383. [CrossRef]

33. Wubuli, A.; Reyer, H.; Muráni, E.; Ponsuksili, S.; Wolf, P.; Oster, M.; Wimmers, K. Tissue-wide gene expression analysis of sodium/phosphate co-transporters in pigs. Int. J. Mol. Sci. 2019, 20, 5576. [CrossRef] [PubMed]

34. Seynaeve, M.; Janssens, G.; Hesta, M.; Van Nevel, C.; De Wilde, R. Effects of dietary Ca/P ratio, P level and microbial phytase supplementation on nutrient digestibilities in growing pigs: Precaecal, post-ileal and total tract disappearances of OM. J. Anim. Physiol. Anim. Nutr. 2000, 83, 36-48. [CrossRef] 